\title{
An Optimized Prefabricated Raft Footing System for Houses on Shrink-Swell Soils: Preliminary Results
}

\author{
Bertrand Teodosio $^{1 *}$, Kasun Shanaka Kristombu Baduge ${ }^{2}$, and Priyan Mendis ${ }^{3}$ \\ ${ }^{1}$ PhD candidate, Department of Infrastructure Engineering, University of Melbourne \\ ${ }^{2}$ Research fellow, Department of Infrastructure Engineering, University of Melbourne \\ ${ }^{3}$ Professor, Department of Infrastructure Engineering, University of Melbourne \\ "Corresponding author's e-mail: b.teodosio@student.unimelb.edu.au
}

\begin{abstract}
The strong demand for houses has been hampered by a shortage of skilled labor in Australia, which can be potentially alleviated using prefabrication. Significant advancements in the design and construction of prefabricated houses have been observed; however, most substructure constructions still use traditional cast-in-place method that is labor intensive and weatherdependent. Prefabrication of footing systems is an advantageous solution since this require minimal manual labor and shorter construction period. The design of an innovative prefabricated footing needs to consider structural integrity and design assembly. One of the important structural issues for light-weight houses is cyclic differential ground movements affecting footing systems due to reactive soils. This shrink-swell movements are due to the decrease and increase in soil moisture, which can cause minor to severe damage depending on the presence of fines. Due to the issues on shortage of skilled labor and housing, and the costly impact of shrink-swell movements of reactive soils to footings, this study aims to develop a prefabricated footing based on optimized waffle raft. The developed system can easily be installed in stable to highly reactive sites, minimizing site disturbance, on-site assembly requirements and maximizing construction speed, quality and sustainability.
\end{abstract}

\section{KEYWORDS}

Prefabricated raft footing system; Reactive soils; Topology optimization; Houses

\section{INTRODUCTION}

The positive Australian economic condition and increase in population have led to a growing demand for houses. The Australian property market for dwellings has seen consistent increases of approximately $3 \%$ per annum since 1970s (Stapledon 2010). The average total number of dwelling commencements from 2001 to present is on average 150,000 per year (Australian Bureau of Statistics, 2017); however, the cumulative housing shortage is still around 220,000 (Cannington, 2017). This strong demand for houses has been hampered by a shortage of skilled labor (Lewis, 2017). Prefabrication, the method of constructing off-site then transporting and assembling on-site, is an advantageous solution since this require minimal manual labor and shorter construction period (Gibb 1999). Moreover, additional benefits such as improved site safety, controlled quality of materials and workmanship, and improved sustainability can be achieved (Lichtenberg 2006). 
Significant advancements in the design and construction of prefabricated residential superstructures have been observed ( Friedman, 1992; Barlow and Ozaki, 2005; Song et al., 2005); contrarily, the design and construction of substructures still use the traditional cast-in-place method that is prone to construction delay and reduced quality due to weather dependence and other in-situ construction factors (Gispert 2016; Nelson 2008). Prefabrication of footing systems has the potential to give significant improvements to existing construction processes, specifically residential projects that are necessary to be completed in a short period of time (Monash University 2017). This will alleviate the growing issues of housing and skilled labor shortage in Australia and could also be applied globally. The design of an innovative prefabricated footing system needs to consider the structural integrity and design assembly of a substructure system (McFarlane and Stehle 2014). One of the significant issues for light-weight houses is the cyclic differential ground movements affecting the footing systems due to reactive soils (Abdelmalak 2007; Briaud et al. 2012). Reactive soils are clay-type soils that significantly change their volume, swelling when soil moisture increased, and shrinking when soil moisture decreased. The damage due to shrink-swell movements of the ground may range from a minor hairline to severe structural cracks requiring major repairs (Appendix C, AS 2870-2011).

Due to the issues on shortage of skilled labor and housing, and the costly impact of shrink-swell movements of reactive soils to footings, this study aims to develop an optimized prefabricated raft footing for single-detached dwellings constructed on stable to highly reactive soils. The prefabricated footing solution shall be structurally robust and can easily be assembled. In turn, this will minimize crack damage, site disturbance, in-situ assembly requirements and waste generation.

\section{METHODS}

This study aims to develop an optimized prefabricated raft footing for houses having robust structural features to minimize the effect of shrink-swell movements of reactive soils. The specific objectives of this study are: (1) to develop an optimized prefabricated raft footing system based on waffle raft suitable for low-rise and light-weight residential structures on reactive soils; (2) to investigate the performance of the developed system using field monitoring and non-destructive crack testing; (3) to compare existing methods by conducting sensitivity analysis for each design process and establish a suitable approach to predict the behavior of the developed system; and (4) to develop a design guideline for the optimized prefabricated waffle footing system through parametric simulations using validated soil-structure interaction model paired with Concrete Damaged Plasticity (CDP) model. This paper focuses on the first specific objective and preliminary comparison between the new optimized shape of waffle rafts against the traditional shape of waffle rafts. The research framework is presented in Figure 1. 


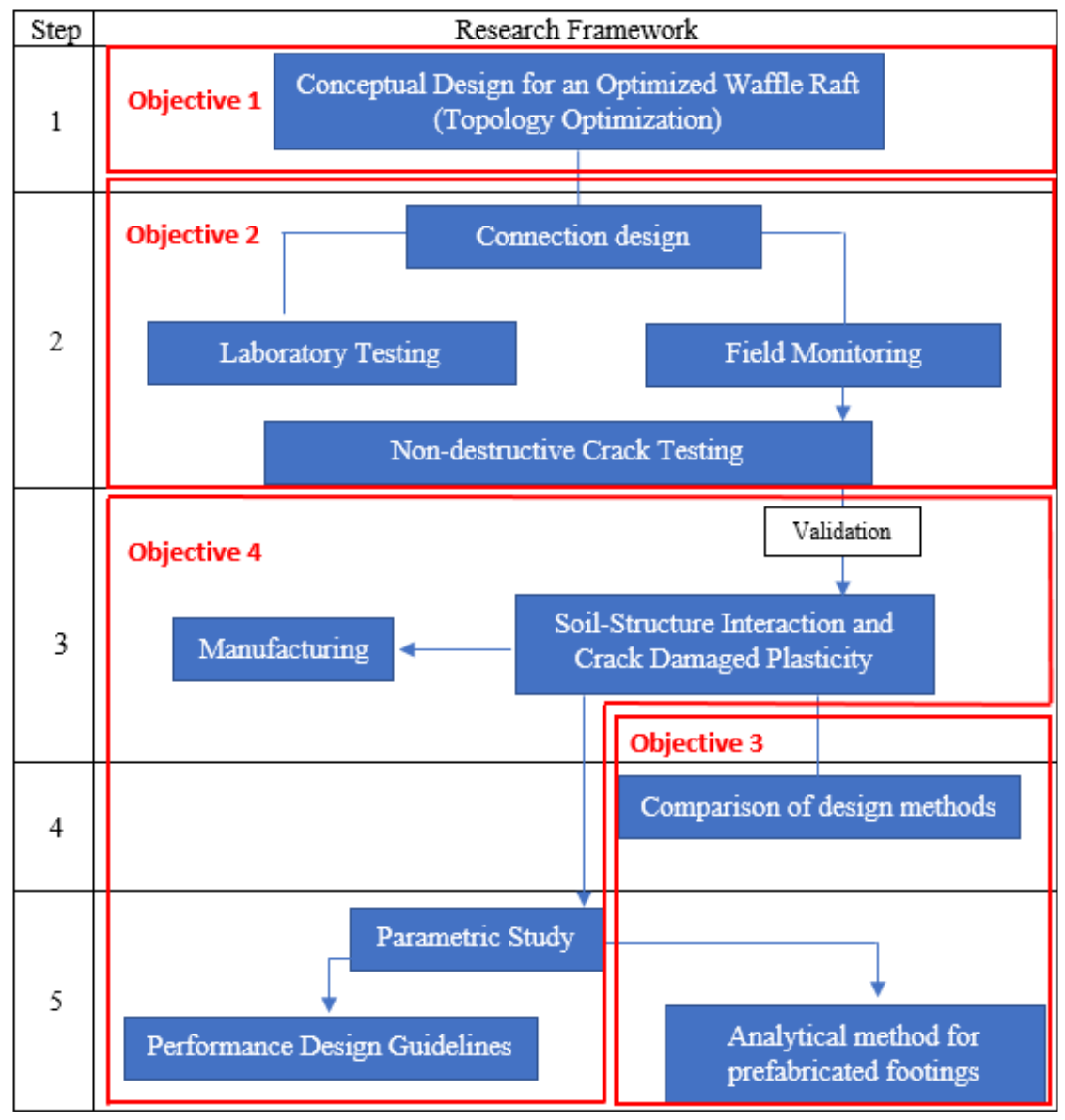

Figure 1. Research framework.

To achieve the primary aim, preliminary investigations were conducted. The methods involved are: (1) topology optimization and (2) preliminary numerical simulations. Topology optimization was used to obtain an optimized conceptual design based on waffle rafts, while preliminary numerical simulations were performed to compare the potential advantages of the shape of the optimized conceptual design against the shape of a conventional waffle raft.

\section{Topology Optimization}

Topology optimization, a mathematical method that optimizes material layout within a given optimization design space depending on a given sets of loads, boundary conditions and other constraints, was used to develop a preliminary conceptual design for a prefabricated footing system (Otomori et al. 2015; Zuo and Xie 2015). An optimized prefabricated footing system based on waffle raft is presented, which can be installed on stable to highly reactive sites, minimizing site disturbance due to shrink-swell movements of reactive soils.

\section{Preliminary Numerical Simulations}

The main goal of the preliminary numerical simulations is to compare the deformation between the optimized raft footing and a conventional waffle raft. The geotechnical and structural features are modelled as three-dimensional finite element instances using Abaqus/CAE. The geotechnical model is comprised of a soil block and a footing system, where the soil reactivity is modelled using 
an elastic porous medium with moisture swelling based on Weerasinghe et al. (2015). The applicability of an elastic model is justified to be acceptable since soils which have undergone wet and dry cycles are expected to have elastic volume changes. These soils are called environmentally stabilized soils (Gould et al. 2011). The structural model is comprised of a footing system subjected to mechanical loads considering structural damage (i.e. Concrete Damaged Plasticity model).

The soil water characteristic curve, given by the plot of negative pore water pressure against degree of saturation ( $-U_{w}$ vs $S_{r}$ ), is necessary to define the sorption behavior of the soil porous medium and an example relationship for $\varepsilon_{m s}$ vs $S_{r}$ by Tripathy et al. (2002) was used for the preliminary simulations. This curve will reflect the change in $-U_{w}$ driven by the soil moisture content increase (wetting) and decrease (drying). An example $-U_{w}$ vs $S_{r}$ curve by Chan et al. (2015) was used. The elastic coefficient, $\kappa$, is assumed to be 0.05 with a Poisson's Ratio value of 0.4 , neglecting tensile limit. The element type for the soil porous medium is an 8-node brick, with trilinear displacement and trilinear pore pressure (Pore Fluid/Stress). A preliminary result for a shrink scenario is presented in Figure 2.

The element type of the footing system is an 8-node linear brick with reduced integration and hourglass control (3D Stress). The footing is a composite structure, in this case a waffle raft to enable us to compare with other existing design methods, combining concrete and steel reinforcements. Since the footing system and the soil porous medium have different mechanical properties, the interaction between the two are defined as contact interaction.

This interaction is critical since it combines the deflection of the footing system due to mechanical loadings (uniform load, $w$, equal to $2.5 \mathrm{kPa}$ and line load, $W$, equal to $6.5 \mathrm{kN} / \mathrm{m}$ ) and the ground movement due to soil moisture changes (change in suction equal to $1.2 \mathrm{pF}$ ). The interaction between the footing and the soil porous medium is comprised of general contact and tangential contact. The frictional coefficient for the tangential contact is 0.25 with friction penalty formulation.

\section{RESULTS AND DISCUSSION}

\section{Topology Optimization: Conceptual Design}

Topology optimization was used to obtain the conceptual design for the optimized prefabricated footing system based on a waffle raft. The conceptual design is not presented in detail due to a non-disclosure agreement with the Australian Research Council - Centre for Advanced Manufacturing of Prefabricated Housing (ARC-CAMP.H) and the collaborating partner company, the Australian Reinforcing Company.

\section{Preliminary Numerical Simulations}

The comparison of the deformation of the optimized raft footing and the conventional waffle raft had obtained significant differences. The deformation experienced for the center heave scenario by the optimized raft footing was $6 \mathrm{~mm}$, while that of the conventional waffle raft was $14 \mathrm{~mm}$ (Figure 2). On the other hand, the deformation experienced for the edge heave scenario by the optimized raft footing was $2 \mathrm{~mm}$, while that of the conventional waffle raft was $10 \mathrm{~mm}$ (Figure 3 ). 
This comparison was only based on shape and the optimized raft footing was considered as castin-place, without considering connections and panel configurations.

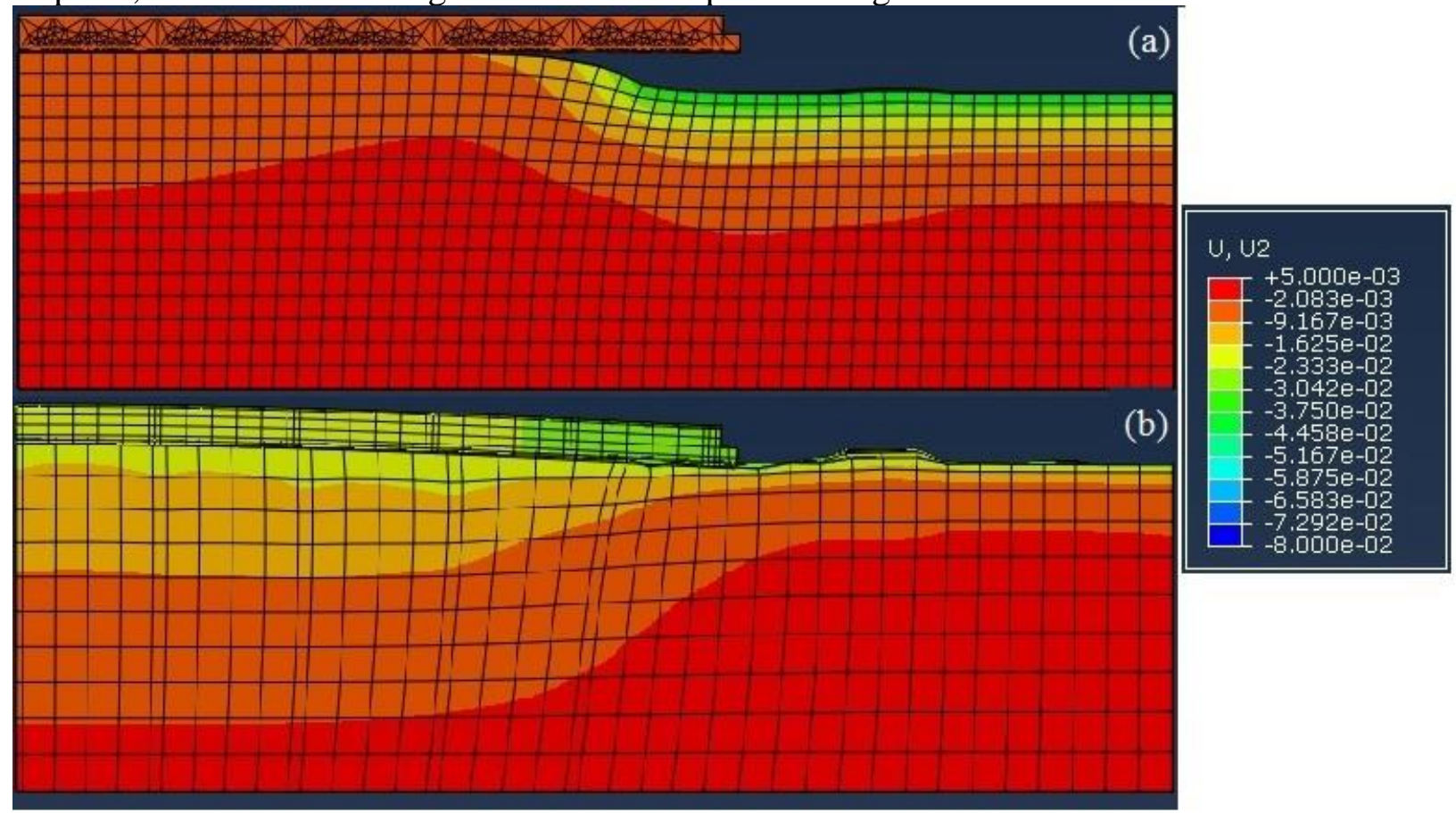

Figure 2. Results of the developed soil-structure interaction model showing the displacements for the center heave scenario of (a) the optimized raft footing and (b) of the conventional waffle raft footing.

The deformation of the optimized raft footing was different to the conventional waffle raft. The optimized raft footing acted as a cantilever for the center heave scenario with an edge penetration distance of $2 \mathrm{~m}$, this behaviour is due to a stiffer section of this footing causing lesser deformation. Although the conventional waffle raft had the same edge penetration distance, this had lesser gap with the soil that reflects a relatively higher magnitude of deflection. Similar findings with the edge heave scenario, the optimized raft footing carried the applied area load effectively compared to the conventional waffle raft. These comparisons show that the optimized raft footing have stiffer section than the conventional waffle raft in both center and edge heave scenario.

Due to the potential of the optimized raft footing, future simulations will be performed to incorporate (1) panelizing for handling and assembly and (2) connection design.

\section{CONCLUSION}

This study had developed an optimized prefabricated raft footing system based on a waffle raft. A design based on an optimized waffle raft was chosen since this is the most common and most economical footing system for houses in Australia, which will increase the possibility for market uptake of the proposed prefabricated footing system. 
MOC SUMMIT / MAY 2019

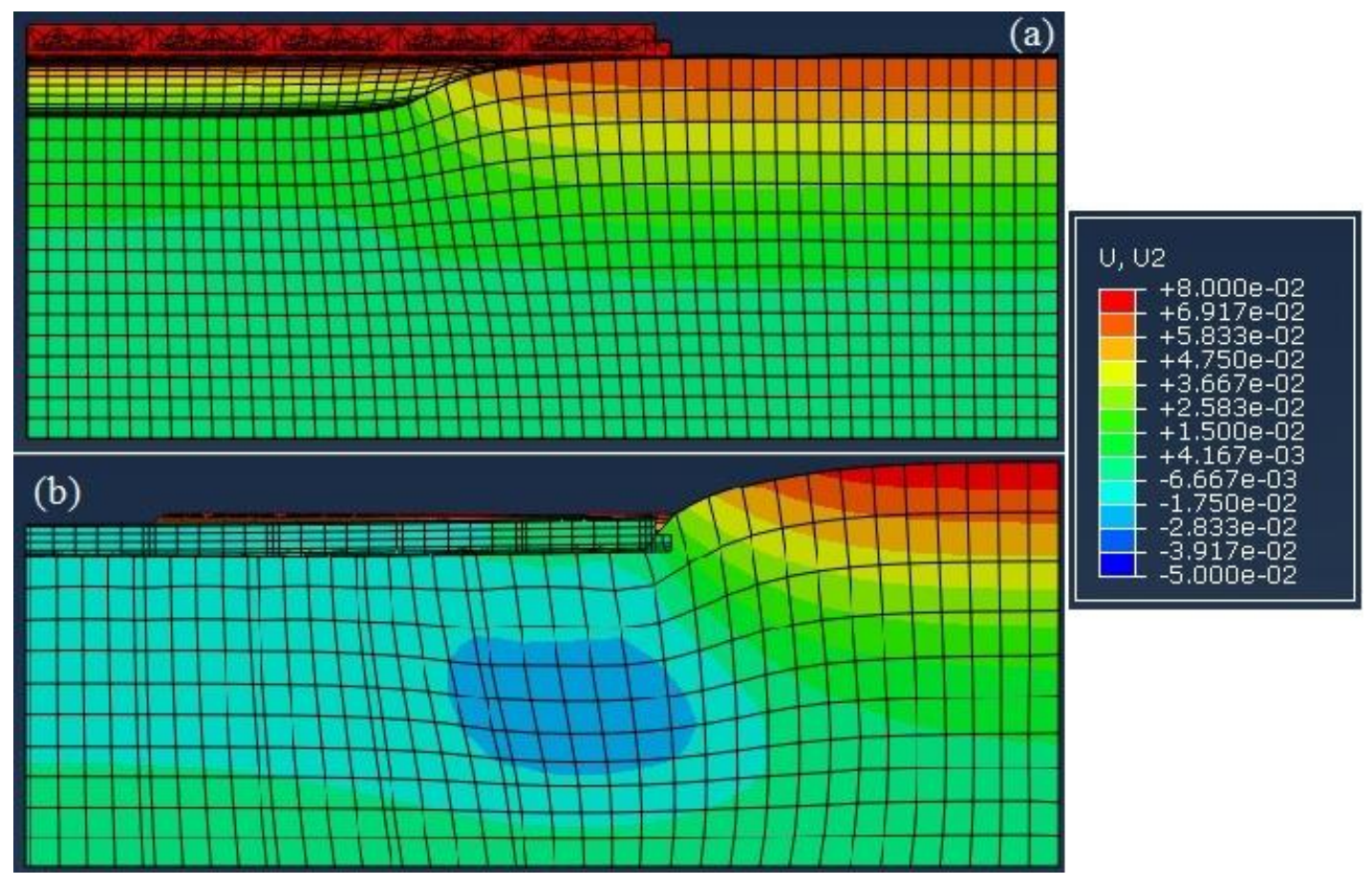

Figure 3. Results of the developed soil-structure interaction model showing the displacements for the edge heave scenario of (a) the optimized raft footing and (b) of the conventional waffle raft footing.

The objective of this paper is to compare the deformation between the optimized raft footing and a conventional waffle raft. The results of the preliminary simulations have obtained significant differences in the magnitude of deformation. The optimized raft footing had significantly lower deformation than the conventional waffle raft. Thus, the optimized shape and features of the developed footing is stiffer than a conventional waffle raft.

To achieve the primary aim and remaining specific objectives of this study, the following future work should be conducted: (1) validation of the performance of the optimized prefabricated footing and the developed model through laboratory experiments and field monitoring, (2) comparison of design methods and formulation of a new design process considering prefabrication; (3) parametric simulations of the validated soil-structure interaction model. One of the important details to be finalized is the connection to be used for the prefabricated panels of the optimized shape of footing.

\section{ACKNOWLEDGEMENTS}

The authors would like to thank the Australian Research Council - Centre for Advanced Manufacturing of Prefabricated Housing (ARC-CAMPH) Project ID IC150100030.

\section{REFERENCES}

Abdelmalak, R. I. (2007). Soil structure interaction for shrink-swell soils "A new design procedure for foundation slabs on shrink-swell soils.” Texas A\&M University. 
Adams, M. W., and Bradley†, P. (1945). "Dimensional Coordination *." Journal of the American Ceramic Society, 28(8), 217-226.

American Society for Testing and Materials. (2002). "Standard Guide for Dimensional Coordination of Rectilinear Building Parts and Sysems."

Australasian Procurement and Construction Council. (2014). Procurement of Construction Products - a guide to achieving compliance.

Australian Bureau of Statistics. (2017). "Australian Bureau of Statistics: Building Approvals." <http://www.abs.gov.au/Ausstats/abs@.nsf/mf/8731.0> (Sep. 6, 2017).

Australian Government. (2011). "Work Health and Safety Act 2011."

Barlow, J., and Ozaki, R. (2005). "Building mass customised housing through innovation in the production system: lessons from Japan." Environment and Planning A, 37(1), 9-20.

Beck, W. G. (1999). "Prefabricated modular concrete foundation wall systems and methods of constructing prefabricated modular concrete foundation wall systems."

Bergvall, L. (1977). "Dimensional coordination as a tool for industrialisation." Metric Dimensional Coordination - The Issue and Precedent.

Brassington, B. (2012). "Code of Practice for Packing of Cargo Transport Units."

Briaud, J. L., Abdelmalak, R., and Zhang, X. (2012). "Design of stiffened slabs-on-grade on shrink-swell soils." Proc. 5th Int. Conf. Unsaturated Soil, International Center for Numerical Methods in Engineering, Barcelona, Spain.

Cannington, D. (2017). Australian Property: Australian Housing Chartbook. 11.

Chan, D., Gallage, C. P. K., Rajeev, P., and Kodikara, J. (2015). "Field performance of in-service cast iron water reticulation pipe buried in reactive clay." Canadian Geotechnical Journal, 52(11), 1861-1873.

Commonwealth. (1995). "Road Transport Reform (Mass and Loading) Regulations."

Commonwealth. (2011). "Heavy Vehicle National Law."

Gibb, A. G. (1999). Off-site fabrication: prefabrication, pre-assembly and modularisation. John Wiley \& Sons.

Gispert, E. (2016). "Prefabricated foundtions for housing applied to room modules."

Gould, S. J., Kodikara, J., Rajeev, P., Zhao, X.-L., and Burn, S. (2011). "A void ratio-water content-net stress model for environmentally stabilized expansive soils." Canadian Geotechnical Journal, 48(6), 867-877.

International Maritime Organization. (2013). "Revised recommendations on harmonized interpretation and implementation of the iternational convention for safe containers (CSC)."

Lewis, K. (2017). "HIA Trades Report September 2017 Quarter.” 6.

Lichtenberg, J. (2006). "Slimbouwen, a strategy for efficient and sustainable building innovation." Proceedings Joint.

McFarlane, A., and Stehle, J. (2014). "DfMA: engineering the future." Proceedings of Council on Tall Buildings and Urban Habitat (CTBUH) 2014 Shanghai Conference, 508-516.

Menegon, S. J., Wilson, J. L., Hughes, S., and Gad, E. F. (2018). "The development of two new innovative precast building core connections." Australasian Structural Engineering Conference, 'ASEC 2018', Adelaide Convention Centre, Adelaide, Australia, 25-28 September 2018.

Monash University. (2017). Handbook for the design of modular structures.

National Transport Commission, National Transport Commission, and Roads and Traffic Authority of New South Wales. (2004). Load restraint guide: guidelines and performance 
standards for the safe carriage of loads on road vehicles. National Transport Commission, Melbourne.

Nelson, S. (2008). "Modular housing: prefab and proudly upmarket." Building Connection, (Summer Quarter 2008), 24.

Otomori, M., Yamada, T., Izui, K., and Nishiwaki, S. (2015). "Matlab code for a level set-based topology optimization method using a reaction diffusion equation." Structural and Multidisciplinary Optimization, 51(5), 1159-1172.

Song, J., Fagerlund, W. R., Haas, C. T., Tatum, C. B., and Vanegas, J. A. (2005). "Considering prework on industrial projects." Journal of construction engineering and management, 131(6), 723-733.

Standards Australia. (1986). AS 1418.1 1986: Cranes, hoists and winches - Part 1 general requirements. Standards Australia, Sydney.

Stapledon, N. (2010). "A History of Housing Prices in Australia 1880-2010."

Tripathy, S., Rao, K. S., and Fredlund, D. G. (2002). "Water content - void ratio swell-shrink paths of compacted expansive soils." Canadian Geotechnical Journal, 39(4), 938-959.

Victorian Legislation. (2004). "Occupational Health and Safety Act 2004."

Victorian Legislation. (2007). "Occupational Health and Safety Regulations 2007."

Weerasinghe, D. R., Kodikara, J., and Bui, H. (2015). "Impact of Seasonal Swell/Shrink Behavior of Soil on Buried Water Pipe Failures." 5.

Zuo, Z. H., and Xie, Y. M. (2015). "A simple and compact Python code for complex 3D topology optimization." Advances in Engineering Software, 85, 1-11. 